Onsager coefficients for a Brownian particle in space-periodic and time-periodic potentials

Peer-reviewed author version

Rosas, Alexandre; VAN DEN BROECK, Christian \& Lindenberg, Katja (2016) Onsager coefficients for a Brownian particle in space-periodic and time-periodic potentials. In: JOURNAL OF PHYSICS A-MATHEMATICAL AND THEORETICAL, 49(48), p. 1-17 (Art No 484001).

DOI: $10.1088 / 1751-8113 / 49 / 48 / 484001$

Handle: http://hdl.handle.net/1942/23983 


\title{
Onsager coefficients for a Brownian particle in space-periodic and time-periodic potentials
}

\author{
Alexandre Rosas* \\ Departamento de Física, CCEN, Universidade Federal da Paraíba, \\ Caixa Postal 5008, 58059-900, João Pessoa, Brazil. \\ Christian Van den Broeck \\ Hassel University, B-3590 Diepenbeek, Belgium. \\ Katja Lindenberg \\ Department of Chemistry and Biochemistry, and BioCircuits Institute, \\ University of California San Diego, La Jolla, California 92093-0340, USA.
}

\begin{abstract}
We perform the thermodynamic analysis of an engine consisting of a Brownian particle in a spaceperiodic and time-periodic potential, including the issues of power, efficiency and dissipation. We derive the explicit expressions for the Onsager coefficients characterizing the linear response regime.
\end{abstract}

\section{INTRODUCTION}

By construction, thermodynamic machines operate in a time-periodic fashion: such a machine actually functions as a catalyst, returning to its initial state after each cycle. The simplest time-periodic modulation corresponds to a steady state operation, i.e., with a continuous translational symmetry in time. Nonequilibrium steady states have in fact been the focus of a large body of research in nonequilibrium statistical mechanics. Famous results include the symmetry of the Onsager coefficients and the fluctuation-dissipation relations linking these coefficients to equilibrium fluctuations. Much less attention has been devoted to genuine time-periodic perturbations, especially from the perspective of a thermodynamic analysis. In fact, the evaluation of Onsager coefficients for such a situation is a recent development [1-10]. By averaging over one period of the perturbation one finds, in the linear response regime, an effective steady state characterized by expressions for the entropy production, the fluxes, and the Onsager coefficients that are similar to those of the traditional nonequilibrium steady states. An important novelty, however, is that the corresponding Onsager coefficients need not be symmetric, because the time reversal symmetry can be broken by the modulation. This leads to a number of questions, in particular concerning the efficiency of such an engine and its relation to power and dissipation [11, 12]. In this context, the relations between the regimes of maximum efficiency, maximum power, and minimum dissipation have recently been clarified [13].

In this contribution we consider a thermodynamic machine consisting of an overdamped Brownian particle in a potential which is periodic both in time and space, and evaluate how energy and entropy flow through this device. The effect of modulated space-periodic potentials on Brownian motion has been the object of a great

*arosas@fisica.ufpb.br deal of research as a prototype model for Brownian motors. The best known examples are the rectification of the Brownian motion in flashing and in rocking ratchets. In this context, the regime of linear response has, however, received scarce attention [14], probably because the rectified transport of particles via Brownian ratchets is a nonlinear effect. Our argument in the present paper is that a Brownian particle in a modulated potential can function as an engine when operating in the linear regime. Furthermore, this operation can, as we will show, be analyzed in full analytic detail. In particular, we derive explicit expressions for the Onsager coefficients characterizing the linear response regime. In our model, the energy flow takes place via the Brownian particle. Hence, the corresponding analysis reveals thermodynamic properties of an isothermal small scale engine, a subject of considerable interest in nano and biotechnology.

Our model is isothermal. We are thus not dealing with a thermal engine, but rather with the transformation of one form of work into another. In the absence of dissipation, such a transformation has a $100 \%$ efficiency. However, the power extracted from such a perfectly efficient machine vanishes. Our explicit results will provide an illustration of their interrelation. An additional benefit of our analysis is that it applies to a small scale system, since the energy flow takes place via the Brownian particle.

This paper is organized as follows. In the next section, we discuss in more detail the thermodynamic set-up. In Sec. III we obtain our main results for the entropy production and, in the linear response regime, the Onsager coefficients. Two simple applications of our theory are discussed in Sec. IV. Finally, in Sec. V we present some concluding remarks.

\section{STATEMENT OF THE PROBLEM}

We assume that a Brownian particle is in contact with a heat reservoir at temperature $T$ and under the influence 
of a periodic potential energy of the form

$$
U(x, t)=U_{0}(x)+Y_{1}(x) \mathcal{F}_{1}(t)+Y_{2}(x) \mathcal{F}_{2}(t)
$$

where $U_{0}(x)$ is a background potential. The functions $Y_{j}(x)$ have spatial period $L$ and the $\mathcal{F}_{j}(t)$ have temporal period $\tau$. The separation into two contributions, $U_{j}(x, t)=Y_{j}(x) \mathcal{F}_{j}(t)$ with $j=1,2$ allows the interpretation of the potential as a thermodynamic machine that transforms work into work, with $j=1$ playing the role of the load (output work) and $j=2$ the role of the driver (input work). Since total energy is conserved, the difference between these two must be the heat dissipated by the particle into the heat bath. It is interesting to explore how these work and heat contributions depend on the details of the machine, such as the shapes and the rates of change of the potentials. On a more technical note, we point out that the separation into the product of functions $Y_{j}(x)$ and $\mathcal{F}_{j}(t)$ is somewhat arbitrary, but the products have the units of energy. For convenience, which will become clear below, we interpret $Y_{j}(x)$ as a displacement (units of distance) and $\mathcal{F}_{j}(t)$ as a force.

In the overdamped limit, the time evolution of a Brownian particle under the influence of such a potential is described by the Langevin equation:

$$
\dot{x}=-\gamma^{-1} \frac{\partial U(x, t)}{\partial x}+\xi(t),
$$

where $\gamma$ is the dissipation parameter and $\xi(t)$ is a Gaussian white noise with zero mean and correlation function

$$
\left\langle\xi(t) \xi\left(t^{\prime}\right)\right\rangle=\frac{k_{B} T}{2 \gamma} \delta\left(t-t^{\prime}\right) .
$$

Here $k_{B}$ is the Boltzmann constant. The Langevin equation can be converted to a Fokker-Planck equation for the probability density $\mathcal{P}(x, t)$ of finding the particle at the position $x$ at time $t$,

$$
\frac{\partial \mathcal{P}}{\partial t}=\hat{W} \mathcal{P}
$$

where the operator $\hat{W}$ is defined as

$$
\hat{W}(x, t)=\gamma^{-1} \frac{\partial}{\partial x} U_{x}(x, t)+D \frac{\partial^{2}}{\partial x^{2}} .
$$

Here $U_{x}(x, t)$ is the derivative of the potential $U(x, t)$ with respect to $x$ and the diffusion coefficient is given by the Einstein relation as

$$
D=\frac{k_{B} T}{\gamma} .
$$

After an initial transient, the system will reach a "timeperiodic and space-periodic steady state" in the sense that, at any given time, the probability of finding the Brownian particle will acquire the symmetry of the potential and be $L$-periodic in space (and $\tau$-periodic in time). Hence, we can define the reduced probability density

$$
P(x, t)=\sum_{k=-\infty}^{\infty} \mathcal{P}(x+k L, t)
$$

which obeys the Fokker-Planck equation Eq. (4) in the interval $[0, L]$ with periodic boundary conditions. From here on, we will drop the description "reduced" and refer to $P(x, t)$ simply as the probability density.

\section{ENTROPY PRODUCTION AND ONSAGER COEFFICIENTS}

Because in the steady state the Brownian particle will acquire the periodicity of the potential, the expected value of the energy of the Brownian particle after a complete cycle will return to its value at the start of the cycle. Consequently, the net work done on the particle as a result of the interplay of the two potentials $U_{1}(x, t)$ and $U_{2}(x, t)$ is completely transformed into heat. The total rate of entropy production over one cycle, which is associated with the rate of the production of heat averaged over one cycle, can here be equally well expressed as the average of the rate of production of work,

$$
\dot{S}=\frac{1}{T} \frac{1}{\tau} \int_{0}^{\tau} \int_{0}^{L} \frac{\partial U(x, t)}{\partial t} P(x, t) d x d t .
$$

The time periodicity of the forces suggests that a natural way to proceed is to expand $\mathcal{F}_{j}(t)$ in a Fourier series,

$$
\mathcal{F}_{j}(t)=\sum_{\mu} F_{\mu}^{(j)} g_{\mu}(t)
$$

where we have introduced the compact notation $\mu=$ $(n, \zeta), n=1,2, \ldots$, representing the Fourier modes (the term $n=0$ can be absorbed into the background potential), and $\zeta=c$ or $s$ such that

$$
\begin{aligned}
& g_{n, c}(t)=\cos \left(\frac{2 \pi n t}{\tau}\right), \\
& g_{n, s}(t)=\sin \left(\frac{2 \pi n t}{\tau}\right) .
\end{aligned}
$$

Using the Fourier expansion, Eq. (8) yields

$$
\begin{aligned}
\dot{S} & =\sum_{\mu} \frac{1}{T} \frac{1}{\tau} \int_{0}^{\tau} \int_{0}^{L} Y_{1}(x) F_{\mu}^{(1)} \dot{g}_{\mu}(t) P(x, t) d x d t \\
& +\sum_{\mu} \frac{1}{T} \frac{1}{\tau} \int_{0}^{\tau} \int_{0}^{L} Y_{2}(x) F_{\mu}^{(2)} \dot{g}_{\mu}(t) P(x, t) d x d t \\
& =\sum_{\mu}\left(X_{\mu}^{(1)} J_{\mu}^{(1)}+X_{\mu}^{(2)} J_{\mu}^{(2)}\right)
\end{aligned}
$$

where we have defined

$$
X_{\mu}^{(j)}=\frac{F_{\mu}^{(j)}}{T},
$$


and the flux associated with $X_{\mu}$,

$$
J_{\mu}^{(j)}=\frac{1}{\tau} \int_{0}^{\tau} \int_{0}^{L} Y_{j}(x) \dot{g}_{\mu}(t) P(x, t) d x d t .
$$

Here we can see that our convention that $\mathcal{F}_{j}(t)$ is a force and $Y_{j}(x)$ is a displacement implies that the flux has the convenient units of speed.

Our results so far are valid regardless of the values of the forces, from weak to strong. To proceed to the linear response regime, which we can handle analytically, we first consider the case of weak forces. We will return to the question of what happens beyond this regime in our examples (Sec. IV). In the linear response regime, the responses (fluxes) are assumed to be proportional to the forces, that is,

$$
J_{\mu}^{(j)}=\sum_{\nu, k} L_{\mu, \nu}^{j, k} X_{\nu}^{(k)}
$$

The Onsager coefficients $L_{\mu, \nu}^{j, k}$ are then given by

$$
L_{\mu, \nu}^{j, k}=\left.\frac{\partial J_{\mu}^{(j)}}{\partial X_{\nu}^{(k)}}\right|_{\mathbf{F}=\mathbf{0}}
$$

where $\mathbf{F}=\mathbf{0}$ indicates that all $F_{\mu}^{(j)}$ are zero.

Next we note that the only dependence of $J_{\mu}^{(j)}$ on $X_{\nu}^{(k)}$ is via the probability density $P(x, t)$ [cf. Eq.(14)],

$$
\frac{\partial J_{\mu}^{(j)}}{\partial X_{\nu}^{(k)}}=\frac{1}{\tau} \int_{0}^{\tau} \int_{0}^{L} Y_{j}(x) \dot{g}_{\mu}(t) \frac{\partial P(x, t)}{\partial X_{\nu}^{(k)}} d x d t
$$

In the Appendix we obtain an expression for $\partial P / \partial X_{\nu}^{(k)}$ and perform the integrals over time. With these steps we finally obtain

$$
\begin{aligned}
& \left.L_{(m, \zeta),\left(n, \zeta^{\prime}\right)}^{j, k}=-(-1)^{\delta_{\zeta, c}} k_{B}^{-1} \frac{\pi n \bar{~} \overline{\left[Y_{j}-\overline{Y_{j}}\right]\left[Y_{k}-\overline{Y_{k}}\right]}\left(1-\delta_{\zeta, \zeta^{\prime}}\right) \delta_{m, n}}{\left(\frac{2 \pi n}{\tau}\right)^{2}+\lambda_{p}^{2}}\right)^{2}\left[-\lambda_{p} \delta_{\zeta, \zeta^{\prime}}+(-1)^{\delta_{\zeta, c}}\left(\frac{2 \pi n}{\tau}\right)\left(1-\delta_{\zeta, \zeta^{\prime}}\right)\right] \\
& \quad+k_{B}^{-1} \sum_{p} \frac{1}{2} \frac{\left(\frac{2 \pi n}{\tau}\left[Y_{j}(x)-\overline{Y_{j}}\right] \psi_{p}(x) d x \int_{0}^{L}\left[Y_{k}(x)-\overline{Y_{k}}\right] \psi_{p}(x) d x\right\} \delta_{m, n} .}{(18)}
\end{aligned}
$$

Here $\delta_{\zeta, \zeta^{\prime}}$ is the Kronecker delta. We use the overbar to indicate an average of any function of $f(x)$ over the stationary state $P_{e q}(x)$,

$$
\bar{f}=\int_{0}^{L} P_{e q}\left(x^{\prime}\right) f\left(x^{\prime}\right) d x^{\prime} .
$$

$\lambda_{p}$ is the $p$-th eigenvalue associated with the corresponding eigenfunction $\psi_{p}(x)$ of the unperturbed Fokker Planck equation:

$$
\hat{W}_{0} \psi_{p}(x)=\lambda_{p} \psi_{p}(x)
$$

with

$$
\hat{W}_{0}=\left.\hat{W}\right|_{\mathbf{F}=\mathbf{0}}=\gamma^{-1} \frac{\partial}{\partial x} U_{0, x}(x)+D \frac{\partial^{2}}{\partial x^{2}} .
$$

$U_{0, x}(x)$ stands for the derivative of the background potential $U_{0}(x)$ in Eq. (1). The eigenfunctions $\psi_{p}(x)$ are supposed to be orthonormalized with respect to the following inner product:

$$
\langle f \mid g\rangle=\int_{0}^{L} \frac{f(x) g(x)}{P_{e q}(x)} d x
$$

while the operator $\hat{W}_{0}$ is symmetric with respect to this product due to detailed balance. The index $p$ runs over all the eigenfunctions with the corresponding eigenvalues ordered in a decreasing way. Hence, $p=0$ corresponds to the equilibrium distribution $\psi_{0}(x)=P_{\mathrm{eq}}(x)$ with eigenvalue $\lambda_{0}=0$ and we have $0>\lambda_{1} \geq \lambda_{2} \geq \lambda_{3} \ldots$ We finally note that the contribution of the ground state in the sum over $p$, i.e. the $p=0$ term in Eq. (18), in fact vanishes.

In the next section we evaluate the Onsager coefficients and the resultant fluxes, entropy production rates, and efficiency for two particular examples and compare some of our results with those of numerical simulations. We choose simple examples that can illustrate the results without adding unnecessary complexity.

\section{PARTICULAR CASES}

\section{A. Single modulated potential}

To explicitly calculate the Onsager coefficients for the case of a single modulated potential, in Eq. (1) we need to 
specify a background potential $U_{0}(x)$, the portion $Y_{1}(x)$ of the potential to be modulated, and the modulation $\mathcal{F}_{1}(t)$. We have set the other modulated contribution, $Y_{2}(x) \mathcal{F}_{2}(t)$, equal to zero. This example simplifies further if we choose the modulated potential $Y_{1}(x)$ to be the ratio of one of the eigenfunctions $\psi_{p}(x)$ of the secular equation
Eq. (20) to the equilibrium distribution, that is,

$$
Y_{1}(x)=L \frac{\psi_{p}(x)}{P_{e q}(x)},
$$

and the time modulation

$$
\mathcal{F}_{1}(t)=F_{c} \cos \left(\frac{2 n \pi t}{\tau}\right)+F_{s} \sin \left(\frac{2 n \pi t}{\tau}\right) .
$$

The prefactor $L$ in Eq. (23) is included in order to preserve our convention that $Y_{j}(x)$ has units of distance. With these definitions, the surviving Onsager coefficients then reduce to the much simpler expressions

$$
\begin{aligned}
& L_{c, c}^{1,1}=L_{s, s}^{1,1}=-\frac{k_{B}^{-1} L^{2}}{2 \tau} \frac{n^{2}\left(L^{2} \lambda_{p} / D\right) \alpha}{n^{2}+(2 \pi)^{-2}\left(L^{2} \lambda_{p} / D\right)^{2} \alpha^{2}} \\
& L_{c, s}^{1,1}=-L_{s, c}^{1,1}=\frac{\pi n k_{B}^{-1} L^{2}}{\tau}\left[1-\frac{n^{2}}{n^{2}+(2 \pi)^{-2}\left(L^{2} \lambda_{p} / D\right)^{2} \alpha^{2}}\right]
\end{aligned}
$$

where the dimensionless variable $\alpha=\tau D / L^{2}$ quantifies the importance of diffusion over one spatial period during one period of oscillation. The simplest choice for the background potential is a constant potential $U_{0}(x)=$ $U_{0}=0$, which can be chosen to be zero. The eigenvalues of $\hat{W}_{0}$ are

$$
\lambda_{p}=-\left(\frac{2 \pi p}{L}\right)^{2} D
$$

with $p=0,1,2, \ldots$ The spectrum is however degenerate, so that there are two corresponding orthonormal eigenfunctions for each value of $p$, which we indicate, for obvious reasons, with the supplementary index $c$ and $s$ :

$$
\begin{aligned}
\psi_{0}(x) & =P_{e q}(x)=\frac{1}{L} \\
\psi_{(p, c)}(x) & =\frac{\sqrt{2}}{L} \cos \left(\frac{2 \pi \kappa x}{L}\right) \\
\psi_{(p, s)}(x) & =\frac{\sqrt{2}}{L} \sin \left(\frac{2 \pi \kappa x}{L}\right) .
\end{aligned}
$$

Note that in this case, the sum over $p$ in Eq. (18) is a sum over all eigenfunctions, hence including the $c$ and $s$ indices.
We performed the numerical integration of Eq. (2) for $10^{5}$ particles (samples) with $Y_{1}(x)$ and $\mathcal{F}_{1}(t)$ given respectively by Eqs. (23) and (24), with $n=1, p=$ $(1, c), L=1$ and $\tau=1$. After a transient of 1000 time steps $\Delta t=10^{-4}$, we recorded the position of each particle with a precision of $10^{-4}$ every 10 time steps. Hence, we were able to calculate $P(x, t)$ with $10^{4}$ bins both in space and time. Then we used this numerically calculated probability distribution density to evaluate the flux $J_{\mu}^{(j)}$ from Eq. (14). A technical note: in order to get better statistics, the simulations were run over three periods of time and the probability distribution density was averaged over them. The results of our simulations are shown in Fig. 1.

The linear growth of the fluxes for small forces (linear response regime) is clearly very well described by our analytic results, as shown in the expansion of this regime (middle of Fig. 1a) shown in Fig. 1b. We stress that there are no fitting parameters in our analytic results. In Fig. 1a, we see that beyond the linear regime, as $|F|$ increases the diagonal fluxes go through a maximum and then decay, eventually saturating, while the off-diagonal fluxes grow monotonically until they saturate.

With the Onsager coefficients result of Eq. (25) we can also calculate the entropy production rate in the linear response regime:

$$
\dot{S}=\sum_{\mu, \nu} X_{\mu}^{(1)} L_{\mu, \nu} X_{\nu}^{(1)}=-k_{B} \frac{\left(F_{c}^{2}+F_{s}^{2}\right) \beta^{2} L^{2}}{2 \tau} \frac{n^{2}\left(\alpha L^{2} \lambda_{p} / D\right)}{n^{2}+(2 \pi)^{-2}\left(\alpha^{2} L^{2} \lambda_{p} / D\right)^{2}} .
$$

In Fig. 2 we show the dependence of the entropy pro- 


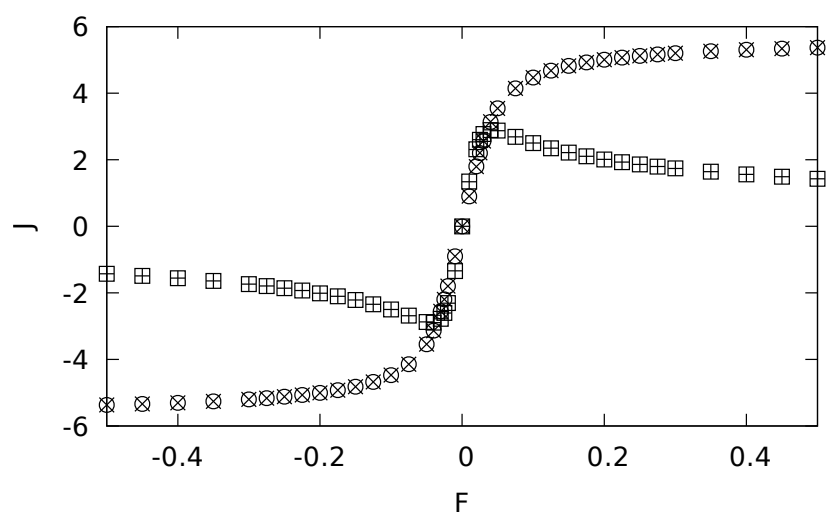

(a) Including large $F$.

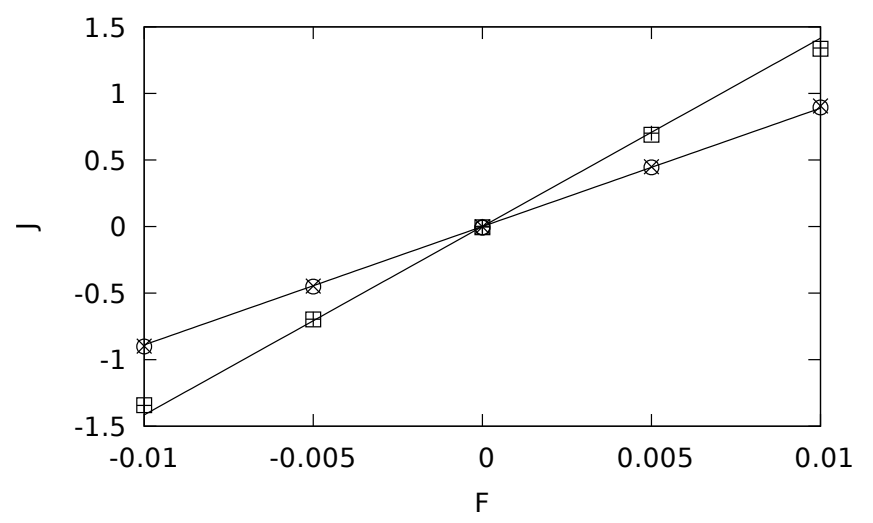

(b) Linear regime. Lines have the slope given by Eqs. (25) divided by $T$.

FIG. 1. Flow $J_{\mu}$ as a function of the $F_{\nu}$. The symbols represent the numerical results: circles $J_{c}$ vs $F_{s}$, crosses $-J_{s}$ vs $F_{c}$, squares $J_{c}$ vs $F_{c}$ and plus signs $J_{s}$ vs $F_{s}$. In the simulations, we set: $L=1, \tau=1, D=0.1$ and $\gamma=0.1$.

background potential, $U_{0}(x)=0$, and with the same parameters as in the previous figure. For small $F$, we see that the quadratic increase of the entropy production predicted by Eq. (30) (linear response regime, see inset) agrees very well with the results of the simulation. For large forces (beyond the linear response regime), as a consequence of the saturation of the diagonal flux, we see that the entropy production rate increases linearly with $F$. We can understand this behavior using a simple argument. When applying a very strong force for a given spatial periodicity $L$, the system is quickly transported during each period from the previous potential minimum (which becomes a maximum) to the new minimum, a distance $L / 2$ away, and that is basically all the dissipation that takes place. Increasing the force will make the drop somewhat larger, $\dot{S} \simeq F * L / 2$. This predicts that the dissipation should become linear in $F$, as observed.

Another striking feature of the entropy production rate in the linear response regime is its dependence on the adimensional parameter $\alpha$, which measures the interplay among the diffusion and the time and space periods of

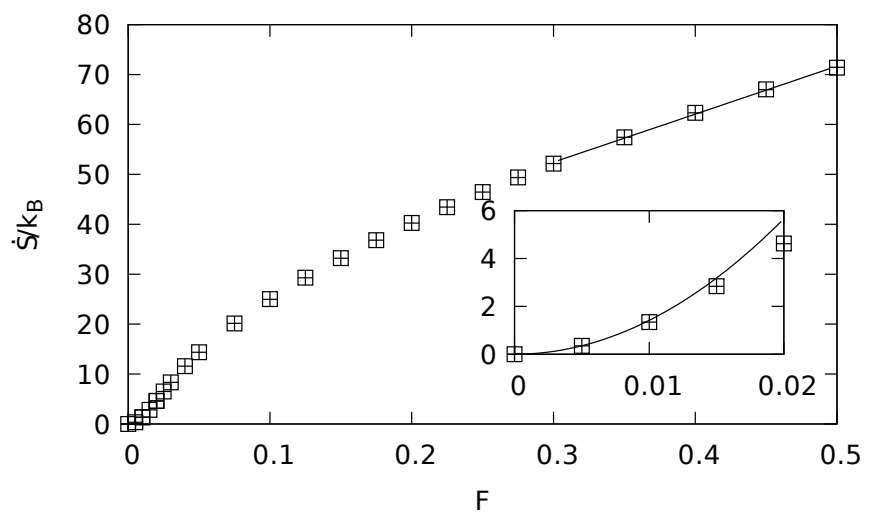

FIG. 2. Entropy production rate as a function of $F$. The line in the main panel represents a linear fit of $\dot{S}$ for large $F$, while in the inset it represents the theoretical prediction Eq (30). The symbols represent the results of the numerical simulations using the same convention of the previous figure. The parameters are $L=1, \tau=1, D=0.1, F_{s}=0$, and $\gamma=0.1$.

the potential. For isothermal processes, for both large and small values of $\alpha$ the entropy production vanishes, but for intermediate values it reaches a maximum (see Fig. 3),

$$
\dot{S}_{\max }=k_{B}\left(F_{c}^{2}+F_{s}^{2}\right) \frac{n \pi \beta^{2} L^{2}}{2 \tau}
$$

at $\alpha=\frac{2 n \pi D}{L^{2}\left|\lambda_{p}\right|}$. Remarkably, the maximum dissipated power (for a single mode) is independent of the spatial mode that defines $U_{1}(x)$. Only the location of the maximum depends on $U_{0}(x)$ and $U_{1}(x)$.

It is interesting to compare the maximum dissipated power $T \dot{S}$ obtained here with the power dissipated by a particle dragged through a fluid of viscosity $\gamma$ by a constant force $F$. The dragged particle will travel with a constant speed $v=F / \gamma$ and dissipate energy per unit time at a rate $v F=F^{2} / \gamma$. Using the Einstein relation Eq. (6), we find that the dissipated power is $F^{2} D / k_{B} T$. In our case, we have a temporally and spatially periodic potential, and consequently a temporally and spatially periodic force. For the sake of comparison, let us consider the simpler case with $F_{s}=0$ in Eq. (24) and calculate the temporal and spatial averages of the square of the force over a uniform probability distribution. In fact, this average should be made using the probability density $P(x, t)$ but for simplicity we use a uniform distribution. Indicating such an average by a bracket, we have that

$$
\begin{aligned}
\left\langle F^{2}\right\rangle & =\left\langle\left(\frac{\partial U}{\partial x}\right)^{2}\right\rangle \\
& =2\left(2 p \pi F_{c}\right)^{2}\left\langle\cos ^{2}\left(\frac{2 n \pi t}{\tau}\right)\right\rangle\left\langle\sin ^{2}\left(\frac{2 p \pi x}{L}\right)\right\rangle \\
& =2\left(p \pi F_{c}\right)^{2}
\end{aligned}
$$


Using this result in Eq. (31), we find that

$$
T \dot{S}_{\max }=\frac{L^{2} n\left\langle F^{2}\right\rangle}{4 p^{2} \pi \tau k_{B} T}=\frac{\left\langle F^{2}\right\rangle D}{2 k_{B} T} .
$$

Therefore, the power dissipated by the Brownian particle

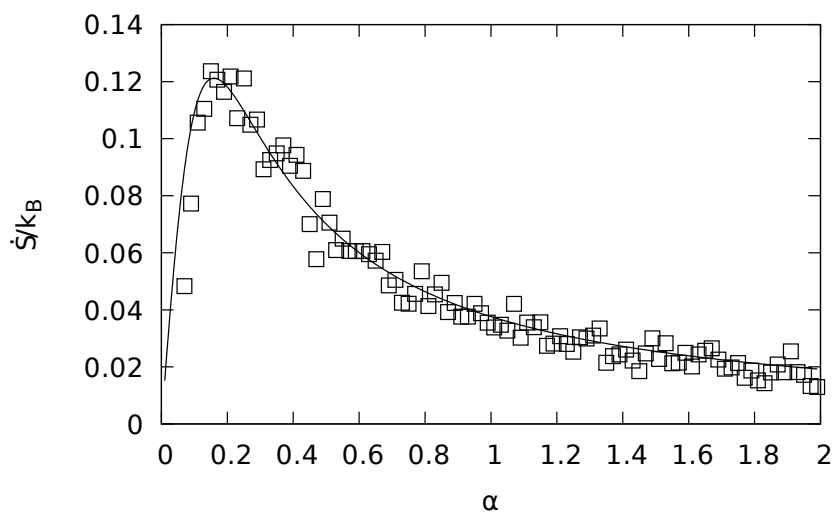

FIG. 3. Entropy production rate for constant temperature. The line represents the theoretical prediction, Eq. (30), while the symbols represent the results of the numerical simulations. The parameters are $L=1, \tau=1, F_{c}=0.05, F_{s}=0$, and $D \gamma=0.18$.

subjected to the modulated potential is similar to the power dissipated by a dragged particle.

We can also ask what happens to the entropy production rate if the viscosity coefficient remains constant but the temperature varies. In this case, we see the monotonic decay shown in Fig. 4, obtained by numerical simulations and also very accurately predicted by our analytic result Eq. (30).

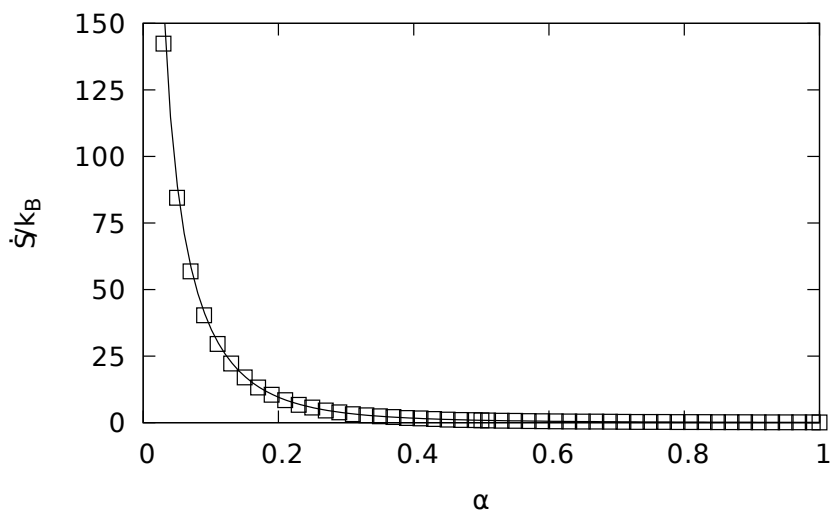

FIG. 4. Entropy production rate as a function of $\alpha$ for constant $\gamma$. The line represents the theoretical prediction Eq (30) while the symbols represent the results of the numerical simulations. The parameters are $L=1, \tau=1, F_{c}=0.05, F_{s}=0$, and $\gamma=0.1$

\section{B. Two modulated potentials with equal spatial configurations: $Y_{1}(x)=Y_{2}(x)$}

If the spatial parts of the two modulated potentials are equal, the Onsager coefficients are related as follows [cf. Eq. (18)]:

$$
L_{(m, \zeta),\left(n, \zeta^{\prime}\right)}^{1,1}=L_{(m, \zeta),\left(n, \zeta^{\prime}\right)}^{2,1}=L_{(m, \zeta),\left(n, \zeta^{\prime}\right)}^{1,2}=L_{(m, \zeta),\left(n, \zeta^{\prime}\right)}^{2,2} .
$$

Moreover, only the same Fourier modes of the modulation functions $\mathcal{F}_{1,2}$ lead to non-vanishing Onsager coefficients. Therefore it is instructive to see what happens to a single mode. Here we will discuss the case where

$$
\begin{aligned}
& \mathcal{F}_{1}(t)=F_{c_{1}} \cos \left(\frac{2 \pi t}{\tau}\right)+F_{s_{1}} \sin \left(\frac{2 \pi t}{\tau}\right), \\
& \mathcal{F}_{2}(t)=F_{c_{2}} \cos \left(\frac{2 \pi t}{\tau}\right)+F_{s_{2}} \sin \left(\frac{2 \pi t}{\tau}\right),
\end{aligned}
$$

and the same (single Fourier mode) spatial potential considered in the previous section, Eq. (23). Hence, we have 16 Onsager coefficients $\left(L_{\zeta, \zeta^{\prime}}^{j, k}\right)$. Nevertheless, most of them are related according to Eq. (34). In fact, all the Onsager coefficients connecting the same modes are equal, $L_{\zeta, \zeta}^{j, k}=L_{c, c}$, and the Onsager coefficients connecting different modes are $L_{c, s}^{j, k}=-L_{s, c}^{j, k}=L_{c, s}$. The values of $L_{c, c}$ and $L_{c, s}$ are the same as in the previous section, cf. Eq. (25). The flux-force relation thus take the following (matrix) form $(J=L X)$,

$$
\left(\begin{array}{c}
J_{c}^{(1)} \\
J_{s}^{(1)} \\
J_{c}^{(2)} \\
J_{s}^{(2)}
\end{array}\right)=\left(\begin{array}{cccc}
L_{c, c} & L_{c, s} & L_{c, c} & L_{c, s} \\
-L_{c, s} & L_{c, c} & -L_{c, s} & L_{c, c} \\
L_{c, c} & L_{c, s} & L_{c, c} & L_{c, s} \\
-L_{c, s} & L_{c, c} & -L_{c, s} & L_{c, c}
\end{array}\right)\left(\begin{array}{c}
X_{c}^{(1)} \\
X_{s}^{(1)} \\
X_{c}^{(2)} \\
X_{s}^{(2)}
\end{array}\right) .
$$

Next we can use this expression to evaluate the entropy production rate,

$$
\dot{S}=X L X,
$$

the power $\mathcal{P}$ extracted from the machine,

$$
\frac{\mathcal{P}}{T}=-X_{c}^{(1)} J_{c}^{(1)}-X_{s}^{(1)} J_{s}^{(1)},
$$

and the efficiency

$$
\eta=-\frac{X_{c}^{(1)} J_{c}^{(1)}+X_{s}^{(1)} J_{s}^{(1)}}{X_{c}^{(2)} J_{c}^{(2)}+X_{s}^{(2)} J_{s}^{(2)}}
$$

From these expressions one can notice that the Fourier components of the forces associated with the potentials $U_{1}(x, t)$ and $U_{2}(x, t)$ must be related so that the power extracted from the machine is in fact positive. This does not necessarily mean that the forces must have opposite signs because we have cross fluxes between cosine and sine modes. However, if only a single Fourier mode (either a sine or a cosine) is available for both potentials, 
it does indeed imply that the forces must have opposite signs.

To find the parameters $X_{c}^{(1)}$ and $X_{s}^{(1)}$ that maximize the power extracted, we set the derivative of $\mathcal{P}$ with respect to those parameters equal to zero and obtain

$$
\begin{aligned}
& X_{c}^{(1)}=-\frac{X_{c}^{(2)} L_{c, c}+L_{c, s} X_{s}^{(2)}}{2 L_{c, c}}, \\
& X_{s}^{(1)}=\frac{X_{c}^{(2)} L_{c, s}-L_{c, c} X_{s}^{(2)}}{2 L_{c, c}},
\end{aligned}
$$

which lead to the following expressions at maximum power:

$$
\begin{aligned}
& \frac{\mathcal{P}_{M P}}{T}=\frac{\left[\left(X_{c}^{(2)}\right)^{2}+\left(X_{s}^{(2)}\right)^{2}\right]\left(L_{c, c}^{2}+L_{c, s}^{2}\right)}{4 L_{c, c}}, \\
& \dot{S}_{M P}=\frac{\left[\left(X_{c}^{(2)}\right)^{2}+\left(X_{s}^{(2)}\right)^{2}\right]\left(L_{c, c}^{2}+L_{c, s}^{2}\right)}{4 L_{c, c}}, \\
& \eta_{M P}=\frac{1}{2}
\end{aligned}
$$

That is, at maximum power half of the power injected is extracted and the other half produces entropy. An interesting observation is apparent concerning the signs of the forces. If $\mathcal{F}_{2}(t)$ has only a single Fourier mode (either the sine or the cosine), the corresponding Fourier mode of $\mathcal{F}_{1}(t)$ will have the opposite sign. A general statement about the sign of the cross Fourier modes is not possible because the flux from a cosine to a sine mode has a sign opposite to the flux from a sine to a cosine mode (the Onsager coefficients $L_{c, s}$ and $L_{s, c}$ have opposite signs).

We might also maximize the efficiency. In this case, we have that

$$
X_{c}^{(1)}=-X_{c}^{(2)}, \quad X_{s}^{(1)}=-X_{s}^{(2)},
$$

leading to

$$
\mathcal{P}_{M \eta}=0, \quad \dot{S}_{M \eta}=0, \quad \eta_{M \eta}=1 .
$$

Consequently, the perfect efficiency scenario can be reached: all the energy inserted in the system is extracted, without entropy production, but this has to be done without extracting any power, as discussed earlier. Furthermore, the conditions given in Eq. (46) imply that $U_{1}(x, t)=-U_{2}(x, t)$ (the forces have opposite signs) so that the net effect of the two periodic potentials on the Brownian particle is zero and no dissipation takes place. The results Eqs.(45)-(47) are in agreement with the general predictions from [13].

A final comment about this particular case of equal spatial modulation functions is that these functions do not change the qualitative behaviour of the system with respect to the maximum power transferred or the maximum efficiency achievable. That is, regardless of the specific form of $U_{0}(x)$ and of $Y_{1}(x)=Y_{2}(x)$, at maximum power half of the power is transferred to the output load and half of it produces entropy, while the maximum efficiency is 1 .

\section{CONCLUDING REMARKS}

We have studied the problem of a Brownian particle under the influence of a unidimensional periodic (in space and time) potential. By separating the time dependent term of the potential into two parts, the Brownian particle can operate as a machine that transfers energy from one work source to the other. We expressed the entropy production in terms of the fluxes and forces acting on the system and, in the linear response regime, obtained explicit expressions for the Onsager coefficients. We illustrated our results in detail in two particular cases. In the case of a single modulated potential, we compared our analytic results with simulations and found very good agreement in the linear response regime. For large forces, the fluxes saturate and the entropy production grows linearly with the forces, a behavior that is easy to explain. Furthermore, we observed that, for a given temperature, the entropy production is maximized for a particular value of the diffusion coefficient. The second example deals with the engine function, by considering two modulated potential contributions, playing the role of driving and load, respectively. We calculated the average power, entropy production and efficiency. In particular, the properties observed in the regimes of maximum power and minimum dissipation, respectively, were found to be in agreement with recent general predictions [13].

\section{Appendix: Onsager coefficients}

As a first step in the evaluation of the Onsager coefficients, we derive the expressions for $\partial P / \partial X_{\nu}^{(k)}$, cf. Eq. (17). In the long time regime, the derivative of the Fokker-Planck equation Eq. (4) at $\mathbf{F}=\mathbf{0}$ in the linear response regime, can be written as:

$$
\frac{\partial P^{(\nu, k)}(x, t)}{\partial t}=\hat{W}^{(\nu, k)}(x, t) P_{e q}(x)+\hat{W}_{0}(x) P^{(\nu, k)}(x, t),
$$

where

$$
\begin{gathered}
P^{(\nu, k)}=\left.\frac{\partial P}{\partial X_{\nu}^{(k)}}\right|_{\mathbf{F}=\mathbf{0}}, \\
\hat{W}^{(\nu, k)}=\left.\frac{\partial \hat{W}}{\partial X_{\nu}^{(k)}}\right|_{\mathbf{F}=\mathbf{0}},
\end{gathered}
$$

and $\hat{W}_{0}$ is given by Eq. (5) at $\mathbf{F}=\mathbf{0}$. The solution of Eq. (A.1) reads:

$$
\begin{aligned}
& P^{(\nu, k)}(x, t)=e^{\hat{W}_{0}(x) t} P^{(\nu, k)}(x, 0) \\
& \quad+\int_{0}^{t} e^{\hat{W}_{0}(x)\left(t-t^{\prime}\right)} \hat{W}^{(\nu, k)}\left(x, t^{\prime}\right) P_{e q}(x) d t^{\prime}
\end{aligned}
$$

The first term on the right hand side is equal to zero because the initial state does not depend on the modulation. As for the second term, we make a change of 
variables, introducing $t^{\prime \prime}=t-t^{\prime}$. Since the eigenvalues of $\hat{W}_{0}$ are negative or zero, while $\hat{W}(x, t) P_{e q}(x)$ is orthogonal to the zero eigenfunction [this follows immediately from the conservation of probability of Eq. (4)], we can extend the limit of integration to infinity:

$$
P^{(\nu, k)}(x, t)=\int_{0}^{\infty} e^{\hat{W}_{0}(x) t^{\prime \prime}} \hat{W}^{(\nu, k)}\left(x, t-t^{\prime \prime}\right) P_{e q}(x) d t^{\prime \prime} .
$$

Now, we define the adiabatic probability distribution $P_{a d}(x, t)$ as the solution, at any time $t$, of

$$
\hat{W} P_{a d}(x, t)=0 \Rightarrow P_{a d}(x, t)=\frac{e^{-\beta U(x, t)}}{Z_{t}},
$$

where $Z_{t}$ is the instantaneous normalization constant :

$$
Z_{t}=\int_{0}^{L} e^{-\beta U(x, t)} d x
$$

and $\beta=1 /\left(k_{B} T\right)$. By taking the derivative of this equation with respect to $X_{\nu}^{(k)}$, and considering the limit of vanishing modulation, we find

$$
\hat{W}^{(\nu, k)}(x, t) P_{e q}(x)+\hat{W}_{0}(x) P_{a d}^{(\nu, k)}(x, t)=0,
$$

which can be used to eliminate the dependence of
Eq. (A.5) on $\hat{W}^{(\nu, k)}$ :

$$
\begin{aligned}
P^{(\nu, k)}(x, t) & =-\int_{0}^{\infty} e^{\hat{W}_{0}(x) t^{\prime \prime}} \hat{W}_{0}(x) P_{a d}^{(\nu, k)}\left(x, t-t^{\prime \prime}\right) d t^{\prime \prime} \\
& =-\int_{0}^{\infty} \frac{d e^{\hat{W}_{0}(x) t^{\prime \prime}}}{d t^{\prime \prime}} P_{a d}^{(\nu, k)}\left(x, t-t^{\prime \prime}\right) d t^{\prime \prime}
\end{aligned}
$$

Next, we integrate Eq. (A.9) by parts:

$$
\begin{aligned}
P^{(\nu, k)}(x, t) & =-\left.e^{\hat{W}_{0}(x) t^{\prime \prime}} P_{a d}^{(\nu, k)}\left(x, t-t^{\prime \prime}\right)\right|_{0} ^{\infty} \\
& -\int_{0}^{\infty} e^{\hat{W}_{0}(x) t^{\prime \prime}} \dot{P}_{a d}^{(\nu, k)}\left(x, t-t^{\prime \prime}\right) d t^{\prime \prime} .
\end{aligned}
$$

The first term vanishes in the limit $t^{\prime \prime} \rightarrow \infty$ (noting that $P_{a d}^{(\nu, k)}$ is orthogonal to the zero eigenfunction of $\left.\hat{W}_{0}\right)$, hence:

$$
P^{(\nu, k)}(x, t)=P_{a d}^{(\nu, k)}(x, t)-\int_{0}^{\infty} e^{\hat{W}_{0}(x) t^{\prime \prime}} \dot{P}_{a d}^{(\nu, k)}\left(x, t-t^{\prime \prime}\right) d t^{\prime \prime} .
$$

We thus find for the Onsager coefficients:

$$
\begin{aligned}
L_{\mu, \nu}^{j, k} & =\frac{1}{\tau} \int_{0}^{\tau} \int_{0}^{L} Y_{j}(x) \dot{g}_{\mu}(t) P^{(\nu, k)}(x, t) d x d t \\
& =\frac{1}{\tau} \int_{0}^{\tau} \int_{0}^{L} Y_{j}(x) \dot{g}_{\mu}(t) P_{a d}^{(\nu, k)}(x, t) d x d t-\frac{1}{\tau} \int_{0}^{\tau} \int_{0}^{L} \int_{0}^{\infty} Y_{j}(x) \dot{g}_{\mu}(t) e^{\hat{W}_{0} t^{\prime}} \dot{P}_{a d}^{(\nu, k)}\left(x, t-t^{\prime}\right) d t^{\prime} d x d t,
\end{aligned}
$$

We next explicitly calculate the derivative of the adiabatic probability density distribution, given in Eq. (A.6):

$$
P_{a d}^{(\nu, k)}(x, t)=-\left.\beta P_{a d}(x, t) \frac{\partial U(x, t)}{\partial X_{\nu}^{(k)}}\right|_{\mathbf{F}=\mathbf{0}}-\left.\frac{1}{Z_{t}} P_{a d}(x, t) \frac{\partial Z_{t}}{\partial X_{\nu}^{(k)}}\right|_{\mathbf{F}=\mathbf{0}}=-k_{B}^{-1} Y_{k}(x) g_{\nu}(t) P_{e q}(x)-\left.\frac{1}{Z_{t}} P_{a d}(x, t) \frac{\partial Z_{t}}{\partial X_{\nu}^{(k)}}\right|_{\mathbf{F}=\mathbf{0}} .
$$

Using the definition of $Z_{t}$, given by Eq. (A.7), we have

$$
\frac{\partial Z}{\partial X_{\nu}^{(k)}}=-k_{B}^{-1} g_{\nu}(t) \int_{0}^{L} e^{-\beta U(x, t)} Y_{k}(x) d x
$$

Hence,

$$
\begin{aligned}
P_{a d}^{(\nu, k)}(x, t) & =-k_{B}^{-1} Y_{k}(x) g_{\nu}(t) P_{e q}(x) \\
& +k_{B}^{-1} P_{e q}(x) g_{\nu}(t) \int_{0}^{L} P_{e q}\left(x^{\prime}\right) Y_{k}\left(x^{\prime}\right) d x^{\prime}
\end{aligned}
$$

Defining $\overline{Y_{k}}$ as the average of the $k$-th displacement function over the equilibrium probability distribution, cf. Eq. (19), in the absence of the modulated potentials, we have:

$$
P_{a d}^{(\nu, k)}(x, t)=k_{B}^{-1} g_{\nu}(t) P_{e q}(x)\left[\overline{Y_{k}}-Y_{k}(x)\right] .
$$

Substituting this expression for $P_{a d}^{(\nu, k)}(x, t)$ in the first integral of Eq. (A.12), we find:

$$
\begin{aligned}
\frac{1}{\tau} \int_{0}^{\tau} \int_{0}^{L} Y_{j}(x) \dot{g}_{\mu}(t) P_{a d}^{(\nu, k)}(x, t) d x d t & =k_{B}^{-1}\left[\frac{1}{\tau} \int_{0}^{\tau} \dot{g}_{\mu}(t) g_{\nu}(t) d t\right]\left[\int_{0}^{L} Y_{j}(x)\left[\overline{Y_{k}}-Y_{k}(x)\right] P_{e q}(x) d x\right] \\
& =k_{B}^{-1}\left[\frac{1}{\tau} \int_{0}^{\tau} \dot{g}_{\mu}(t) g_{\nu}(t) d t\right]\left[\overline{Y_{j}} \overline{Y_{k}}-\overline{Y_{j} Y_{k}}\right]
\end{aligned}
$$


The remaining temporal integral vanishes if the frequencies of the Fourier modes $\mu$ and $\nu$ are different. For $\mu=\nu$, the only contributions are off-diagonal terms, which are anti-symmetric. More precisely, writing $\mu=(m, \zeta)$ and $\nu=\left(n, \zeta^{\prime}\right)$, we have:

$$
\begin{aligned}
& \frac{1}{\tau} \int_{0}^{\tau} \int_{0}^{L} U_{j}(x) \dot{g}_{\mu}(t) P_{a d}^{(\nu, k)}(x, t) d x d t \\
& =(-1)^{\delta_{\zeta, c}} k_{B}^{-1} \frac{\pi n}{\tau}\left[\overline{Y_{j}} \overline{Y_{k}}-\overline{Y_{j} Y_{k}}\right]\left(1-\delta_{\zeta, \zeta^{\prime}}\right) \delta_{m, n} \\
& =-(-1)^{\delta_{\zeta, c}} k_{B}^{-1} \frac{\pi n}{\tau} \overline{\left[Y_{j}-\overline{Y_{j}}\right]\left[Y_{k}-\overline{Y_{k}}\right]}\left(1-\delta_{\zeta, \zeta^{\prime}}\right) \delta_{m, n}
\end{aligned}
$$

We now turn our attention to the remaining integral in Eq. (A.12), which, after substituting the expression for $P_{a d}^{(\nu, k)}$ given by Eq. (A.15), reads

$$
\begin{aligned}
-\frac{1}{\tau} \int_{0}^{\tau} & \int_{0}^{L} \int_{0}^{\infty} Y_{j}(x) \dot{g}_{\mu}(t) e^{\hat{W}_{0} t^{\prime}} k_{B}^{-1} \\
& \times \dot{g}_{\nu}\left(t-t^{\prime}\right) P_{e q}(x)\left[\overline{Y_{k}}-Y_{k}(x)\right] d x d t d t^{\prime} \\
& =-k_{B}^{-1} \frac{1}{\tau} \int_{0}^{\tau} \dot{g}_{\mu}(t) \int_{0}^{\infty} \dot{g}_{\nu}\left(t-t^{\prime}\right) d t^{\prime} d t \\
& \times \int_{0}^{L} Y_{j}(x) e^{\hat{W}_{0} t^{\prime}} P_{e q}(x)\left[\overline{Y_{k}}-Y_{k}(x)\right] d x
\end{aligned}
$$

At this point we introduce the set of eigenvectors of the operator $\hat{W}_{0}$, given by the equation

$$
\hat{W}_{0}\left|\psi_{p}\right\rangle=\lambda_{p}\left|\psi_{p}\right\rangle \text {. }
$$

Here, we assume that the operator $\hat{W}_{0}$ obeys detailed balance as it describes a system at equilibrium in the absence of the time-periodic modulation. Hence the operator is self-adjoint with respect to the following inner product:

$$
\langle f \mid g\rangle=\int_{0}^{L} \frac{f(x) g(x)}{P_{e q}(x)} d x .
$$

Its eigenvalues $\lambda_{p}$ are real (and non-positive) and we can choose the eigenfunctions $\psi_{p}$ to be real. $p=0$ corresponds to the equilibrium state, $\lambda_{p}=0$ and $\psi_{0}(x)=$ $P_{e q}(x)$. The eigenfunctions form a complete orthonormal set, hence one can write:

$$
\begin{aligned}
\left\langle f\left|e^{\hat{W}_{0} t}\right| g\right\rangle & =\left\langle f\left|\left(\sum_{p}\left|\psi_{p}\right\rangle\left\langle\psi_{p}\right|\right) e^{\hat{W}_{0} t}\right| g\right\rangle \\
& =\sum_{p}\left\langle f \mid \psi_{p}\right\rangle\left\langle\psi_{p}\left|e^{\hat{W}_{0} t}\right| g\right\rangle \\
& =\sum_{p}\left\langle f \mid \psi_{p}\right\rangle\left\langle\psi_{p}\left|e^{\lambda_{p} t}\right| g\right\rangle \\
& =\sum_{p} \int_{0}^{L} \frac{f(x) \psi_{p}(x)}{P_{e q}(x)} d x \int_{0}^{L} \frac{\psi_{p}\left(x^{\prime}\right) g\left(x^{\prime}\right) e^{\lambda_{p} t}}{P_{e q}\left(x^{\prime}\right)} d x^{\prime} .
\end{aligned}
$$

Applying this to Eq. (A.18), we can write this integral as:

$$
\sum_{p} \int_{0}^{L} Y_{j}(x) \psi_{p}(x) d x \int_{0}^{L} \psi_{p}\left(x^{\prime}\right) e^{\lambda_{p} t^{\prime}}\left[\overline{Y_{k}}-Y_{k}\left(x^{\prime}\right)\right] d x^{\prime}
$$

Combining the results of Eqs. (A.17), (A.18) and (A.22), the Onsager coefficients of Eq. (A.12) with $\mu=$ $(m, \zeta)$ and $\nu=\left(n, \zeta^{\prime}\right)$ become

$$
\begin{aligned}
L_{(m, \zeta),\left(n, \zeta^{\prime}\right)}^{j, k} & =(-1)^{\delta_{\zeta, c}} k_{B}^{-1} \frac{\pi n}{\tau}\left(\overline{Y_{j}} \overline{Y_{k}}-\overline{Y_{j} Y_{k}}\right)\left(1-\delta_{\zeta, \zeta^{\prime}}\right) \delta_{m, n} \\
& -k_{B}^{-1} \sum_{p} \frac{1}{\tau} \int_{0}^{\tau} \dot{g}_{\mu}(t) \int_{0}^{\infty} e^{\lambda_{p} t^{\prime}} \dot{g}_{\nu}\left(t-t^{\prime}\right) d t^{\prime} d t \\
& \times \int_{0}^{L} Y_{j}(x) \psi_{p}(x) d x \int_{0}^{L} \psi_{p}\left(x^{\prime}\right)\left[\overline{Y_{k}}-Y_{k}\left(x^{\prime}\right)\right] d x^{\prime} .
\end{aligned}
$$

We can rewrite this expression in a more symmetrical form noticing that

$$
\begin{aligned}
\int_{0}^{L} \psi_{p}(x) \overline{Y_{j}} d x & =\overline{Y_{j}} \int_{0}^{L} \frac{\psi_{p}(x) P_{e q}(x)}{P_{e q}(x)} d x \\
& =\left\langle\psi_{p} \mid P_{e q}\right\rangle .
\end{aligned}
$$

Since $\left|P_{e q}\right\rangle=\left|\psi_{0}\right\rangle$, we have that this integral is zero for all excited states. For the ground state, we have that

$$
\int_{0}^{L} P_{e q}(x)\left[\overline{Y_{j}}-Y_{j}(x)\right] d x=\overline{Y_{j}}-\overline{Y_{j}}=0,
$$

so that we can rewrite the Onsager coefficients as

$$
\begin{aligned}
& L_{(m, \zeta),\left(n, \zeta^{\prime}\right)}^{j, k}=-(-1)^{\delta_{\zeta, c}} k_{B}^{-1} \frac{\pi n}{\tau} \overline{\left[Y_{j}-\overline{Y_{j}}\right]\left[Y_{k}-\overline{Y_{k}}\right]}\left(1-\delta_{\zeta, \zeta^{\prime}}\right) \delta_{m, n} \\
& \quad+k_{B}^{-1} \sum_{p \neq 0} \frac{1}{\tau} \int_{0}^{\tau} \dot{g}_{\mu}(t) \int_{0}^{\infty} e^{\lambda_{p} t^{\prime}} \dot{g}_{\nu}\left(t-t^{\prime}\right) d t^{\prime} d t \\
& \quad \times \int_{0}^{L}\left[Y_{j}(x)-\overline{Y_{j}}\right] \psi_{p}(x) d x \int_{0}^{L}\left[Y_{k}\left(x^{\prime}\right)-\overline{Y_{k}}\right] \psi_{p}\left(x^{\prime}\right) d x^{\prime} \delta_{m, n} .
\end{aligned}
$$

To actually perform the integral over $t^{\prime}$ we need to know if the Fourier mode we are analysing is a cosine or a sine. For $\nu=(n, c)$, we have

$$
\begin{aligned}
& -\left(\frac{2 \pi n}{\tau}\right) \int_{0}^{\infty} e^{\lambda_{p} t^{\prime}} \sin \left(\frac{2 \pi n\left(t-t^{\prime}\right)}{\tau}\right) d t^{\prime} \\
& =\left(\frac{2 \pi n}{\tau}\right) \frac{\left(\frac{2 \pi n}{\tau}\right) \cos \left(\frac{2 \pi n t}{\tau}\right)+\lambda_{p} \sin \left(\frac{2 \pi n t}{\tau}\right)}{\left(\frac{2 \pi n}{\tau}\right)^{2}+\lambda_{p}^{2}}
\end{aligned}
$$


while for $\nu=(n, s)$ we have,

$$
\begin{aligned}
& \left(\frac{2 \pi n}{\tau}\right) \int_{0}^{\infty} e^{\lambda_{p} t^{\prime}} \cos \left(\frac{2 \pi n\left(t-t^{\prime}\right)}{\tau}\right) d t^{\prime} \\
& =\left(\frac{2 \pi n}{\tau}\right) \frac{-\lambda_{p} \cos \left(\frac{2 \pi n t}{\tau}\right)+\left(\frac{2 \pi n}{\tau}\right) \sin \left(\frac{2 \pi n t}{\tau}\right)}{\left(\frac{2 \pi n}{\tau}\right)^{2}+\lambda_{p}^{2}}
\end{aligned}
$$

On the other hand, from Eq. (A.12) we see that we need to multiply Eq. (A.27) and Eq. (A.28) by $\dot{g}_{\mu}(t) / \tau$ and integrate over one period. Clearly, only the same frequency mode will give a non-zero result. Moreover, it is easy to see that if both $\mu$ and $\nu$ are sines or cosines, the result of the integral will be

$$
-\frac{1}{2} \frac{\left(\frac{2 \pi n}{\tau}\right)^{2} \lambda_{p}}{\left(\frac{2 \pi n}{\tau}\right)^{2}+\lambda_{p}^{2}},
$$

while the off-diagonal terms are equal to $[\zeta=c$ if $\mu=$ $(n, c)$ and $\zeta=s$ if $\mu=(n, s)]$

$$
(-1)^{\delta_{\zeta, c}} \frac{1}{2} \frac{\left(\frac{2 \pi n}{\tau}\right)^{3}}{\left(\frac{2 \pi n}{\tau}\right)^{2}+\lambda_{p}^{2}} .
$$

Finally, substituting the result of the time integrals Eqs. (A.29) and (A.30) in Eq. (A.26), we obtain the expression for the Onsager coefficients given by Eq. (18).

\section{ACKNOWLEDGMENTS}

AR acknowledges Capes for its support (No. 99999.000296/2015-05). CVdB acknowledges support from UHasselt grant BOF15KVU01. KL acknowledges the support of the US Office of Naval Research (ONR) under Grant No. N00014-13-1-0205.
[1] S. Schmiedl and U Seifert 2008 Europhys. Lett. 8120003.

[2] Y. Izumida and K. Okuda 2009 Phys. Rev. E 80021121.

[3] Y. Izumida and K. Okuda 2010 Eur. Phys. J. B 77499.

[4] M. Esposito, R. Kawai, K. Lindenberg and C. Van den Broeck 2010 Phys. Rev. E 81041106.

[5] Y. Izumida and K. Okuda 2012 Europhys. Lett. 9710004.

[6] Y. Izumida and K. Okuda 2015 New J. Phys. 17085011.

[7] K. Brandner, K. Saito and U. Seifert 2015 Phys. Rev. X 5031019.

[8] K. Proesmans and C. Van den Broeck, Phys. Rev. Lett. 115, 090601 (2015).
[9] K. Proesmans, B. Cleuren and C. Van den Broeck, J. Stat. Mech. 023202 (2016).

[10] K. Proesmans, Y. Dreher, M. Gavrilov, J. Bechhoefer, and C. Van den Broeck, http://arxiv.org/abs/1607.04388.

[11] G. Benenti, K. Saito, and G. Casati, Phys. Rev. Lett. 106, 230602 (2011).

[12] K. Brandner, K. Saito, and U. Seifert, Phys. Rev. Lett. 110, 070603 (2013).

[13] K. Proesmans, B. Cleuren, and C. Van den Broeck, Phys. Rev. Lett. 116, 220601 (2016).

[14] P. Reimann, Physics Reports 361, 57 (2002).

[15] H. Risken, The Fokker-Plank Equation, (Springer-Verlag, 2nd edition, 1989). 